\title{
Wer eHealth sucht, findet einen Haufen Papier
}

\author{
Sima Djalali \\ Dr. med., Institut für Hausarztmedizin, Universität Zürich/UniversitätsSpital Zürich
}

\begin{abstract}
Erstmals seit 2007 wurde in der Schweiz eine repräsentative Befragung von niedergelassenen Ärztinnen und Ärzten zur Computernutzung durchgeführt. Der Anteil von Nutzern elektronischer Krankengeschichten (eKG) hat zugenommen, aber die Analyse von alltäglichen Praxisprozessen zeigt, dass Bits und Bytes das Papier heute noch lange nicht verdrängt haben.
\end{abstract}

Diskussionen um eHealth im Praxisalltag mussten in Ermangelung repräsentativer Daten bislang auf dem Boden der ersten Studie zum "Status quo der IT-Nutzung in Schweizer Arztpraxen" (SISA) aus dem Jahr 2007 geführt werden [2]*. Damals wurde festgestellt, dass 11,7\% der niedergelassenen Ärztinnen und Ärzte eine vollwertige elektronische Krankengeschichte (eKG) nutzten, während 84,3\% zwar einen Praxiscomputer, jedoch ohne volle eKG-Funktionalität nutzten, d.h. rein für administrative Zwecke. Komplett papierbasiert arbeiteten 3,5\%. Angesichts der zwischenzeitlich stattgefundenen Hard- und Software-Evolution und des (standes-)politischen Aufschwungs des Themas eHealth war es an der Zeit, den Wissensstand zu aktualisieren. Daher führte das Institut für Hausarzt-

Qui cherche la cybersanté ne trouvera que du papier

En mai 2013, I'Institut pour la médecine de famille de l'Université de Zurich a effectué une enquête représentative auprès des médecins en libre pratique afin de dresser un état des lieux de l'implémentation des systèmes informatiques et de leur utilisation dans les cabinets médicaux. Les résultats détaillés ont été mis à la disposition de I'Institut pour l'informatique au cabinet médical (IPI) et publiés dans I'International Journal of Medical Informatics [1]. L'on remarquera ainsi que l'implémentation du dossier électronique du patient a augmenté de $23,5 \%$ depuis 2007 et que ce taux se monte désormais à $35,2 \%$. La plupart des médecins qui I'utilisent sont relativement jeunes (30-49 ans) et exercent dans des cabinets de groupe en Suisse alémanique. Ceux-ci n'ont en revanche que rarement fait usage des fonctions informatiques complexes pour la gestion électronique des données, d'informations et de documents. L'échange de données avec d'autres médecins traitants s'est principalement effectué par courrier, fax ou téléphone et rarement par courriel ou d'autres portails en ligne. On constate néanmoins encore des lacunes dans la saisie structurée des données et la pression liée aux exigences freine toujours l'instauration d'un échange à large échelle des données par voie électronique. medizin im Mai 2013 eine erneute Befragung von 1200 niedergelassenen Ärztinnen und Ärzten aller Fachrichtungen durch, mit dem Ziel, den Stand der Implementierung von Praxisinformationssystemen und deren Nutzung zu untersuchen. Die detaillierten Ergebnisse der wissenschaftlichen Auswertung sind aktuell im International Journal of Medical Informatics erschienen [1]. Im Folgenden sollen die wichtigsten Erkenntnisse für die heimischen Leser zusammengefasst werden.

\section{Repräsentative Stichprobe}

Die Adressaten wurden auf Zufallsbasis aus dem FMHAdressregister ausgewählt und erhielten einen Fragebogen per Post zugesandt. Der Rücklauf der Umfrage war mit 57,1\% ausserordentlich hoch, was auf ein ausgeprägtes Interesse an dem Thema schliessen lässt. Die Antworten von 667 (55,6\%) konnten in die Analyse einbezogen werden. Die Zusammensetzung der Teilnehmer hinsichtlich Alter, Geschlecht, Facharzttätigkeit, Praxisform und Niederlassung in der Deutsch- oder Westschweiz resp. im Tessin entsprach den Erwartungswerten gemäss der zu dem Zeitpunkt aktuellen FMH-Statistik [3]. Daher wird die Stichprobe als repräsentativ angesehen. Nicht nur das unterscheidet die aktuelle Studie von anderen Erhebungen $\mathrm{zu}$ dem Thema, die seit 2007 durchgeführt wurden [4-8].

\section{eHealth, das Phantom}

Die Besonderheit der Umfrage war, dass berücksichtigt wurde, dass es keine eindeutige Nomenklatur im eHealth-Bereich gibt. Fragen wie «Nutzen Sie eine eKG?» können Befragte leicht verwirren, weil jeder andere Funktionen damit assoziiert. Auch Verwechslungen 


\begin{tabular}{|c|c|c|c|}
\hline & eKG-Nutzer & $\begin{array}{l}\text { Teilnehmer } \\
\text { mit Computer } \\
\text { ohne volle eKG }\end{array}$ & $\begin{array}{l}\text { Teilnehme } \\
\text { ohne } \\
\text { Computer }\end{array}$ \\
\hline Gesamt & $35,2 \%$ & $59,1 \%$ & $4,5 \%$ \\
\hline \multicolumn{4}{|l|}{ Subgruppen } \\
\hline \multicolumn{4}{|l|}{ Alter } \\
\hline 30-39 Jahre & $73,5 \%$ & $26,5 \%$ & $0,0 \%$ \\
\hline 40-49 Jahre & $55,4 \%$ & $43,4 \%$ & $0,6 \%$ \\
\hline 50-59 Jahre & $31,1 \%$ & $62,3 \%$ & $3,9 \%$ \\
\hline 60-69 Jahre & $19,5 \%$ & $73,0 \%$ & $7,5 \%$ \\
\hline 70-79 Jahre & $13,3 \%$ & $70,7 \%$ & $16,7 \%$ \\
\hline \multicolumn{4}{|l|}{ Geschlecht } \\
\hline Frauen & $36,0 \%$ & $53,7 \%$ & $7,4 \%$ \\
\hline Männer & $35,1 \%$ & $61,4 \%$ & $3,0 \%$ \\
\hline \multicolumn{4}{|l|}{ Sprachregion } \\
\hline Deutsch & $39,9 \%$ & $55,7 \%$ & $3,6 \%$ \\
\hline Französisch & $24,3 \%$ & $66,7 \%$ & $6,8 \%$ \\
\hline Italienisch & $18,8 \%$ & $75,0 \%$ & $6,3 \%$ \\
\hline \multicolumn{4}{|l|}{ Medizinische Fachgruppe } \\
\hline Hausärzte & $44,8 \%$ & $54,1 \%$ & $0,7 \%$ \\
\hline Psychiater & $17,5 \%$ & $63,2 \%$ & $18,4 \%$ \\
\hline Gynäkologen & $26,9 \%$ & $67,3 \%$ & $5,8 \%$ \\
\hline Pädiater & $26,5 \%$ & $70,6 \%$ & $0,0 \%$ \\
\hline andere Spezialärzte & $36,4 \%$ & $59,2 \%$ & $1,6 \%$ \\
\hline \multicolumn{4}{|l|}{ Praxistyp } \\
\hline Einzelpraxis & $26,6 \%$ & $66,6 \%$ & $6,2 \%$ \\
\hline Gruppenpraxis & $44,3 \%$ & $51,4 \%$ & $2,5 \%$ \\
\hline \multicolumn{4}{|l|}{ Dauer der Niederlassung } \\
\hline 0-5 Jahre & $66,7 \%$ & $31,1 \%$ & $0,0 \%$ \\
\hline 6-10 Jahre & $44,1 \%$ & $54,9 \%$ & $1,0 \%$ \\
\hline 11-15 Jahre & $23,7 \%$ & $69,1 \%$ & $5,2 \%$ \\
\hline 16-20 Jahre & $24,4 \%$ & $64,2 \%$ & $7,4 \%$ \\
\hline$>20$ Jahre & $20,5 \%$ & $71,9 \%$ & $7,1 \%$ \\
\hline
\end{tabular}

1 Die Summen der drei Gruppen ergeben nicht notwendigerweise $100 \%$ aufgrund von fehlenden Angaben der Teilnehmer im Bereich von 0-3\% je Frage.

mit dem «Elektronischen Patientendossier» (EPD) liegen nahe. Um Missverständnisse durch Phantombegriffe auszuschliessen, wurden konkrete Arbeitsschritte in der Administration und medizinischen Dokumentation des Praxisalltags abgefragt, z.B. wie die Anamnese schriftlich festgehalten wird, wie Untersuchungen (Labor, verschiedene Arten der Bildgebung usw.) verordnet, empfangen und archiviert werden. Bei elektronischen Arbeitsweisen wurde zudem zwischen strukturierten Daten und unstrukturierten Freitexteinträgen differenziert.

\section{Aufwind für die elektronische Krankengeschichte}

Die aktuelle Anzahl der eKG-Nutzer wurde ermittelt, indem ausgewertet wurde, wie viele Teilnehmer angaben, über einen Computer im Konsultationsraum zu verfügen und die Anamnese und den aktuellen Status des Patienten sowie mindestens Medikamente, Impfungen und/oder Allergien computergestützt zu dokumentieren. Unter Anwendung dieser Diskriminierungskriterien wurde festgestellt, dass 35,2\% der niedergelassenen Ärztinnen und Ärzte eine eKG nutzten. 59,1\% verfügten zwar über einen Computer in der Praxis, jedoch nur für administrative Zwecke, und 4,5\% besassen keinen Praxis-Computer. Diesbezüglich wurden signifikante Unterschiede zwischen Teilnehmern unterschiedlichen Alters, Geschlechts, Sprachregion, Facharztgruppen, Praxistypen und Niederlassungsdauer festgestellt (Tab. 1). Unter 30-49-jährigen, männlichen, in der Deutschschweiz niedergelassenen, als Hausarzt arbeitenden, in Gruppenpraxen tätigen resp. seit maximal 10 Jahren niedergelassenen Teilnehmern war der Anteil der eKG-Nutzer jeweils am höchsten.

\section{Papier bleibt stark}

Bemerkenswert ist, dass administrative IT-Funktionen wie Agendaführung (49,3\%) und TARMED-Erfassung/ Rechnungsstellung $(75, \%)$ weitaus häufiger angegeben wurden, als die Verarbeitung medizinisch relevanter Daten. Laborverordnungen an praxisinterne und externe Labors wurden nur von rund 17\% der Befragten routinemässig über elektronische Formulare verordnet. Der Empfang der entsprechenden Resultate von eigenen Laborgeräten oder externen Labors erfolgte in den wenigsten Praxen (rund 14\% in Bezug auf interne Laborergebnisse, rund 26\% in Bezug auf externe Laborergebnisse) ausschliesslich durch einen strukturierten Datenaustausch. Die Mehrheit der Befragten regelte dies über Brief und Fax und archivierte die Befunde auch in Papierform - selbst wenn sie eine eKG nutzten (Tab. 2). Ähnliche Diskrepanzen zwischen eKG-Nut-

\section{Administrative IT-Funktionen werden weitaus} häufiger per Computer erfasst als medizinisch relevante Daten.

zung einerseits und dem Persistieren papierbasierter Prozesse andererseits, zeigten sich bei der Dokumentation und Archivierung von apparativen Diagnostikergebnissen und Befunden externer Behandler.

Eigene Arztbriefe wurden von 96,3\% der Teilnehmer mit Vorliebe als Brief oder Fax versendet. Immerhin $46,2 \%$ gaben an, gelegentlich (d.h. für rund $10-20 \%$ der ausgehenden Dokumente) auch E-Mails und/oder Online-Dokumentenaustausch-Plattformen für den Versand zu nutzen. Die Zusammenschau aller Angaben zum Handling von Datenein- und -ausgängen legt die 


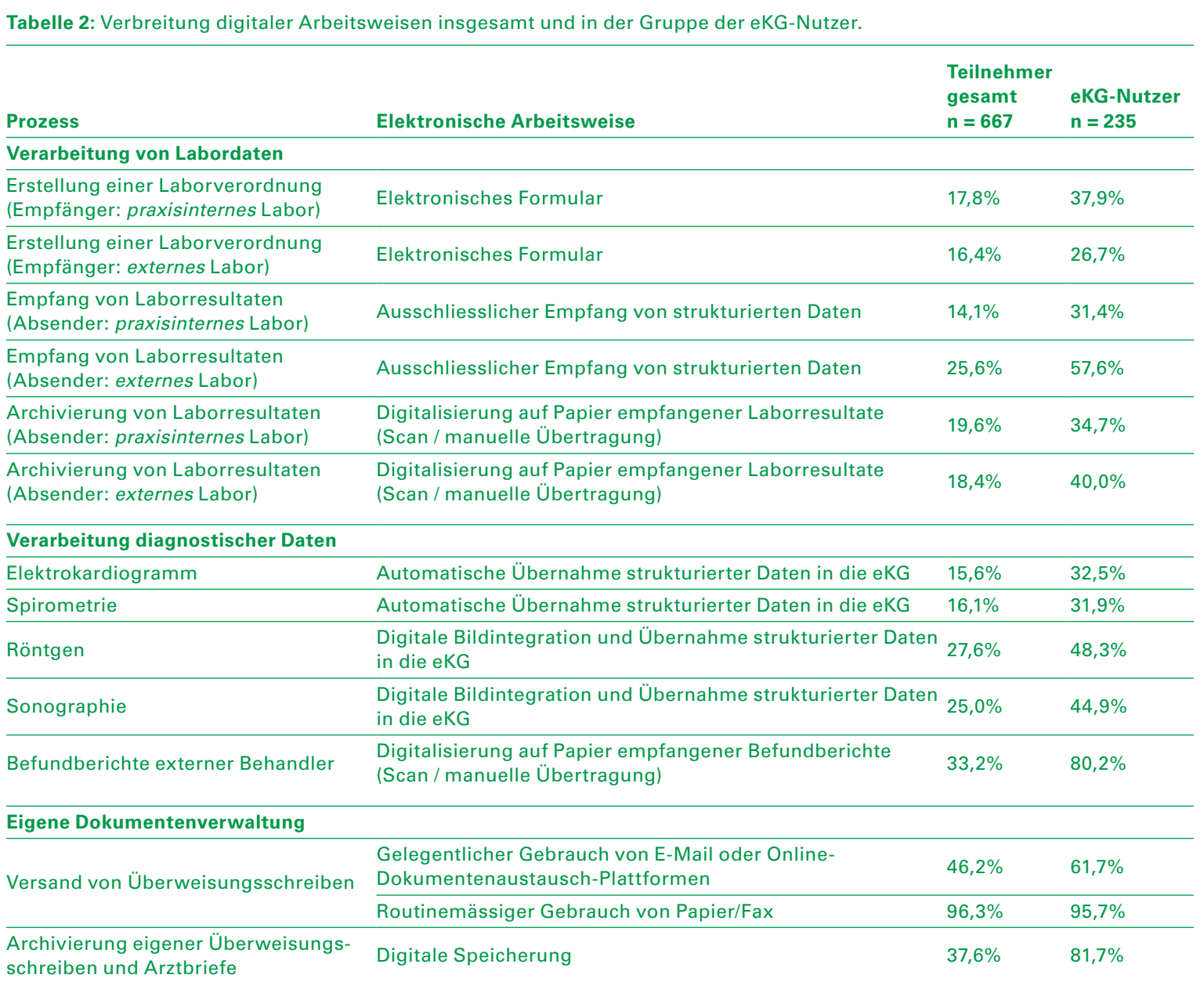

Schlussfolgerung nahe, dass der Anteil von komplett papierlos funktionierenden Praxen in der Schweiz aktuell verschwindend gering resp. quasi nicht existent ist.

\section{Patchwork-Prozesse}

So war der Anteil von Teilnehmern, die komplett elektronische Prozessketten in der Laborverwaltung vorzuweisen hatten, mit rund 3\% sehr gering. Der Anteil von Teilnehmern, die angaben, Befundberichte ausschliesslich elektronisch zu erhalten, war mit $<2 \%$ noch geringer. Teilnehmer, die angegeben hatten, externe Laborergebnisse ausschliesslich bzw. teilweise in elektronischer Form zu erhalten, gaben zwar auch signifikant häufiger an, Befundberichte ausschliesslich oder teilweise elektronisch zu empfangen, doch Teilnehmer, die das gesamte abgefragte Spektrum des Laborund Dokumentenmanagements vollelektronisch und medienbruchfrei organisierten, konnten nicht identifiziert werden.

Selbst Ärzte, die eKGs, elektronischen Laborwertempfang und Mailaustausch von Dokumenten nutzen, kamen also offenbar nicht ohne papierbasierte Arbeitsweisen aus. Sei es, weil Schnittstellen zu anderen digitalen Systemen schlicht fehlen oder weil ihr Umfeld von Empfängern und Sendern ihnen weiterhin den Austausch von Papierdokumenten aufzwingt, deren nachträgliche Digitalisierung möglicherweise unpraktischer ist als das Führen einer parallelen papierbasierten Ablage.

\section{Lust auf mehr?}

Die Mehrheit der Befragten (66,7\%) gab an, nicht die Absicht zu haben, ihren gegenwärtigen Grad der IT-Nutzung innerhalb der nächsten drei Jahre zu verstärken und elektronische Arbeitsprozesse in der Praxis auszubauen. eKG-Nutzer waren signifikant häufger gewillt, weitere Arbeitsprozesse zu digitalisieren als Teilnehmer ohne eKG. Die generell positivere Grundeinstellung von eKG-Nutzern gegenüber elektronischen Arbeitsweisen zeigte sich auch in einer Detailbefragung. Die Teilnehmer gaben an, wie zuversichtlich sie sind, dass die Umstellung auf elektronische Arbeitsweisen zeit- und kosteneffizient sei, die 
Zusammenarbeit mit anderen Behandlern erleichtere, die Qualität der Behandlung verbessere, Arbeitsabläufe unterstütze, komplexe Patientensituationen erfassbar mache resp. Einfluss auf die Arzt-PatientenBeziehung nehme.

\section{Meinung spielt eine untergeordnete Rolle}

Die multivariable Analyse des Zusammenhangs zwischen Meinungsbild und tatsächlicher Übernahme strukturierter elektronischer Arbeitsweisen ergab, dass die Meinung zwar durchaus eine Rolle spielte (Asso-

Der Anteil von komplett papierlos funktionierenden Praxen in der Schweiz ist aktuell verschwindend gering.

ziation zwischen negativer Meinung und Bevorzugung papierbasierter Arbeitsweisen resp. zwischen positiver Meinung und Übernahme elektronischer Arbeitsweisen), verglichen mit dem Einfluss von Arbeitsplatzfaktoren (Gruppen- vs. Einzelpraxis, Sprachregion, Facharztgruppe) jedoch einen geringen Effekt hatte.

\section{Fazit}

Daraus sind folgende Schlüsse zu ziehen: 1. Reine Image-Kampagnen für eHealth sind wahrscheinlich nicht ausreichend, um die Nutzung und den Austausch strukturierter elektronischer Daten unter niedergelassenen Ärzten in der Schweiz in Zukunft zu steigern. 2. Solange nicht eine kritische Masse von elektronisch arbeitenden Ärzten erreicht ist, die einen gegenseitigen Gruppendruck auslösen, werden «Patchwork»-Arbeitsweisen persistieren, bei denen dieselbe Praxis mit unterschiedlichen Einrichtungen unterschiedliche Wege des Datenaustauschs pflegt und bei der Archivierung der Daten/Informationen den Weg des (zumindest kurzfristig gesehen) geringsten Aufwands geht. Solange von den Austauschpartnern vornehmlich der papierbasierte Austausch bevorzugt wird, wird vermutlich auch die papierbasierte resp. doppelte Archivierung bevorzugt werden und die Nachfrage nach Schnittstellen für einen strukturierten Datenaustausch gering sein.

Dr. med. Sima Djala

Institut für Hausarzt-

medizin

UniversitätsSpital Zürich

Pestalozzistrasse 24

CH-8091 Zürich

Tel. 0442557504

sima.djalali[at]usz.ch group" gerechnet werden kann, lässt sich mit der vorliegenden Querschnittsstudie nicht beantworten. Der Vergleich der SISA-I-Studie 2007 und der aktuellen SISA-II-Studie ergab, dass die eKG-Abdeckung zwischen 2007 und 2013 um 23,5\% zugenommen hat. Aber SISA-II zeigt auch eindrücklich, dass eKG-Verfügbarkeit und Fähigkeit zum elektronischen Datenaustausch zwei verschiedene Angelegenheiten sind. Eine lineare Entwicklung anzunehmen, ist aufgrund der Fülle von Einflussfaktoren sowohl bei der eKG-Implementierung als auch beim elektronischen Daten-, Informations- und Dokumentenaustausch unrealistisch.

\section{Eingreifen}

Fest steht, dass tatsächlich mit dem häufig postulierten, aber auch gelegentlich hinterfragten «Generationeneffekt» zu rechnen ist [9-12]. Die Theorie besagt, dass Generationen, die bereits in jungem Alter den alltäglichen Einsatz digitaler Medien erlebt haben - sogenannte «Digital Natives» - diese Technologien besser beherrschen und darum auch in der ärztlichen Tätigkeit selbstverständlich nutzen werden.

Die Tatsache, dass jüngere, kürzer niedergelassene Teilnehmer in der vorliegenden Studie in Bezug auf fast alle abgefragten Szenarien häufiger angaben, elektronische (wenn möglich auf strukturierten Daten basierende) Arbeitsweisen zu nutzen, positiv zu bewerten und in den nächsten drei Jahren weiter ausbauen

Die Mehrheit gab an, ihren gegenwärtigen Grad der IT-Nutzung innerhalb der nächsten drei Jahre nicht verstärken zu wollen.

zu wollen, bestätigt die Existenz des Generationeneffekts. Dies spricht dafür, dass die besagte kritische Masse von elektronisch arbeitenden Austauschpartnern durch nachrückende, stärker IT-affine Ärztegenerationen früher oder später erreicht werden wird. Die Frage ist, ob legislative oder finanzielle Eingriffe die Entwicklung beschleunigen könnten.

\section{Anmerkung}

Die Studie wurde finanziell vom Institut für Praxisinformatik (IPI) unterstützt. Die FMH unterstützte die Arbeit durch die Verfügbarmachung einer Zufallsstichprobe von Adressen niedergelassener Ärzte. IPI und FMH hatten zu keinem Zeitpunkt Einfluss auf die Datenerhebung, -analyse und Publikation der Arbeit. 


\section{Literatur}

1 Djalali S, Ursprung N, Rosemann T, Senn O, Tandjung R. Undirected health IT implementation in ambulatory care favors paper-based workarounds and limits health data exchange. International Journal of Medical Informatics. 2015;15(84):920-32.

DOI: 10.1016/j.ijmedinf.2015.08.001

2 Rosemann T, Marty F, Bhend $\mathrm{H}$ et al. Utilisation of information technologies in ambulatory care in Switzerland. Swiss Medical Weekly. 2010;140:w13088

3 Kraft E. FMH-Ärztestatistik 2011. 30849 Ärztinnen und Ärzte für die Schweiz. Schweiz Ärztezeitung. 2012;93(11):399-403.

4 Golder L, Beer C, Tschöpe S et al. Schlussbericht Swiss eHealth Barometer 2013. Studie im Auftrag der Info Society Days. gfs Bern; 2013.

5 Golder L, Beer C, Tschöpe S et al. Schlussbericht Swiss eHealth Barometer 2014. Studie im Auftrag der Info Society Days. gfs Bern; 2014.

6 Golder L, Bieri U, Imfeld M et al. Schlussbericht Swiss eHealth Barometer 2012. Studie im Auftrag der Info Society Days. gfs Bern; 2012.
7 Djalali S, Bhend H, Rosemann T. [Electronic medical record in Swiss primary care-discrepancy between expectations and reality]. Praxis. 2013;102:641-6.

8 Schoen C, Osborn R, Squires D et al. A survey of primary care doctors in ten countries shows progress in use of health information technology, less in other areas. Health affairs. 2012;31:2805-16

9 Carr F. Doctors, EHR. Can This Shotgun Marriage Be Saved? Information Week 2014. www.informationweek.com/healthcare/ electronic-health-records/doctors-and-ehr-can-this-shotgunmarriage-be-saved/d/d-id/1113763; Accessed July 30, 2014

10 Martin J. Digital doctoring. New York Times 2007. www.nytimes. com/2007/04/10/opinion/10iht-edmartin.1.5212649.html Accessed July 30, 2014

11 Martins M. New physicians prefer the digital experience. The Health Experience Project 2014; http://thehealthexperienceproject.com/ new-physicians-prefer-digital-experience/; Accessed July 30, 2014.

12 Vartabedian B. The digital physician. 33 charts - medicine health (social) media 2011; http://33charts.com/2011/10/digital-physician. html Accessed July 30, 2014. 